\title{
Food insecurity and its associations with bulimic-spectrum eating disorders, mood disorders, and anxiety disorders in a nationally representative sample of U.S. adults
}

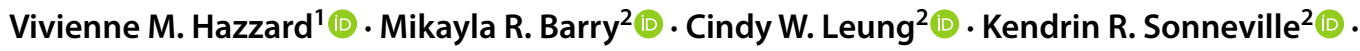 \\ Stephen A. Wonderlich ${ }^{1} \cdot$ Ross D. Crosby ${ }^{1}$
}

Received: 16 October 2020 / Accepted: 22 June 2021 / Published online: 27 June 2021

(c) Springer-Verlag GmbH Germany, part of Springer Nature 2021

\begin{abstract}
Purpose To examine cross-sectional associations between food insecurity and 12-month eating disorders, mood disorders, and anxiety disorders among U.S. adults.

Methods This study used data collected between 2001 and 2003 from 2914 participants in the National Comorbidity SurveyReplication, a nationally representative sample of U.S. adults (mean age $=44.9$ years; $53.4 \%$ female). Twelve-month food insecurity was assessed with a modified version of the Short Form U.S. Household Food Security Scale. Twelve-month DSM-IV diagnoses of mental disorders were based on the World Health Organization Composite International Diagnostic Interview. Modified Poisson regression models were conducted, adjusting for age, sex, race/ethnicity, education, and incometo-poverty ratio.

Results Food insecurity was experienced by $11.1 \%$ of participants. Food insecurity was associated with greater prevalence of bulimic-spectrum eating disorders (prevalence ratio $[\mathrm{PR}]=3.81$; 95\% confidence interval $[\mathrm{CI}] 2.26-6.42$ ), mood disorders ( $\mathrm{PR}=2.53$; 95\% CI 1.96-3.29), and anxiety disorders ( $\mathrm{PR}=1.69 ; 95 \% \mathrm{CI} 1.39-2.07)$.

Conclusion Results indicate that food insecurity is associated with a range of internalizing mental disorders, though these findings should be confirmed with contemporary data to reflect DSM-5 diagnostic updates and the economic effects of the COVID-19 pandemic. Findings from this study emphasize the need to expand food insecurity interventions and improve access to mental health services for food-insecure populations.
\end{abstract}

Keywords Food insecurity $\cdot$ Eating disorders $\cdot$ Mood disorders $\cdot$ Depressive disorders $\cdot$ Anxiety disorders

\section{Introduction}

Food insecurity—defined as limited or uncertain means to access nutritious food in a safe and socially acceptable manner [1] - is a social determinant of health that affected over one in ten households in the United States (U.S.) in 2019 [2] and has affected many more in 2020 amidst the COVID-19 pandemic [3]. Possibly related to lower dietary quality [4], food insecurity is associated with a range of

Vivienne M. Hazzard

viviennehazzard@gmail.com

1 Sanford Center for Biobehavioral Research, 120 Eighth Street South, Fargo, ND 58103, USA

2 Department of Nutritional Sciences, University of Michigan School of Public Health, Ann Arbor, MI, USA adverse physical health outcomes, including type 2 diabetes and hypertension [5]. Food insecurity is also associated with poorer mental health outcomes, including depression and anxiety [6-11], which may arise from the feelings of helplessness, shame, and stress that qualitative studies suggest are common in the experience of food insecurity [12, 13]. Additionally, an emerging body of evidence suggests that food insecurity may be associated with eating disorders [14-17], which are highly comorbid with mood and anxiety disorders [18]. Disrupted eating patterns are characteristic of both food insecurity and eating disorders and, therefore, represent an important, albeit understudied, parallel between the two phenomena.

Eating disorders, which include, among others, anorexia nervosa, bulimia nervosa, and binge-eating disorder, are estimated to affect nearly $9 \%$ of individuals in the United States at some point in their lives [19]. In addition 
to commonly co-occurring with other psychiatric disorders, they are associated with substantial psychosocial impairment, medical complications, and suicidality [18, 20, 21]. Therefore, given their prevalence and burden of disease, eating disorders represent a significant public health concern. While eating disorders were historically believed to occur most commonly in affluent populations, eating disorders are now recognized to affect individuals across all socioeconomic strata [22]. Moreover, in recent years, individuals living in food-insecure households have demonstrated higher levels of overall eating disorder pathology [14, 15], more eating disorder symptoms such as binge eating [14, $15,23,24]$ and compensatory behaviors (e.g., self-induced vomiting, laxative use) $[14,15,25]$, and greater likelihood of meeting diagnostic criteria for bulimia nervosa [17] and binge-eating disorder [16] as assessed via self-administered questionnaire. However, no studies to date have examined the association between food insecurity and eating disorders using eating disorder data gleaned via diagnostic interview, which offers important advantages over self-administered questionnaire data (e.g., the opportunity for interviewers to probe for thoughtful responses [26]), and most prior studies have utilized convenience samples. Furthermore, to our knowledge, only one study thus far-conducted among adolescents [9] — has used diagnostic interview data from a national sample to examine food insecurity in relation to mood and anxiety disorders. Therefore, the objective of this study was to examine cross-sectional associations between food insecurity and 12-month diagnoses of eating disorders, mood disorders, and anxiety disorders as assessed via diagnostic interview in the National Comorbidity Survey Replication (NCS-R), a nationally representative sample of U.S. adults. While NCS-R data were collected two decades ago in 2001-2003, the NCS-R is still regarded as one of the two best nationally representative population-based sources of U.S. eating disorder prevalence estimates derived via diagnostic interview available to date [19]. The otherthe National Epidemiologic Survey on Alcohol and Related Conditions-III (NESARC-III) [27]—is more recent but did not assess food insecurity. We hypothesized that the prevalence of eating disorders, mood disorders, and anxiety disorders would each be greater among food-insecure individuals than among food-secure individuals.

\section{Methods}

\section{Participants}

The NCS-R is a nationally representative survey of the U.S. household population administered in-person to Englishspeaking adults between February 2001 and April 2003 [28, 29]. The sample was based on a multi-stage clustered area probability design; the response rate was 70.9\% [29]. The survey was administered in two parts. Part I was administered to all participants $(N=9282)$ to assess for mood disorders, anxiety disorders, substance use disorders, and impulse control disorders. Part II was administered to all participants who met lifetime criteria for any of the disorders assessed in Part I of the survey in addition to a probability sample of other participants $(N=5692)$. Part II assessed potential correlates, as well as other mental disorders. Eating disorders were assessed in a subsample of participants randomly selected from those who completed Part II $(N=2980)$. The Human Subjects Committees of both Harvard Medical School and the University of Michigan approved all study procedures [29]. This study has thus been performed in accordance with the ethical standards laid down in the 1964 Declaration of Helsinki and its later amendments.

The present study used data from the Part II subsample of the NCS-R who completed assessment for eating disorders. In this subsample of 2980 participants, missing rates were $2 \%$ or lower for each variable of interest in this study. Participants with missing data on any variables of interest were excluded from analyses, resulting in an analytic sample of 2914 participants.

\section{Measures}

\section{Food security}

Twelve-month food security was assessed in the NCS-R with five items drawn and modified from the Short Form U.S. Household Food Security Survey Module (HFSSM) [30]. In this modified version, which has been utilized in other publications using NCS data [9], skip logic was introduced and two items were combined into the following single item, "How many months in the past 12 months did you either cut the size of your meals or skip meals because there wasn't enough money to buy food?" (participants could respond with any integer between 0 and 12). These modifications were made to reduce participant burden within the context of the full interview, which ranged from 90 min to $6 \mathrm{~h}$ depending upon the complexity of psychiatric history [29]. The modified measure used in the NCS-R is provided in Online Resource 1. As in the original Short Form HFSSM, the number of affirmative responses was summed for a score ranging from 0 to 6 and then dichotomized, with two or more affirmative responses indicating food insecurity [30]. Among U.S. households, a cut-point of two or more affirmative responses on the original Short Form HFSSM has a $92.0 \%$ sensitivity and $99.4 \%$ specificity for detecting food insecurity as assessed by the 18-item HFSSM, which is considered the gold standard measure of food security status in the United States [30]. 


\section{Diagnostic assessment}

Diagnoses were based on the version of the World Health Organization (WHO) Composite International Diagnostic Interview (CIDI) developed for the World Mental Health (WMH) Survey Initiative [26]. Twelve-month eating disorder (anorexia nervosa, bulimia nervosa, and binge-eating disorder), mood disorder (major depressive disorder, dysthymia, bipolar I disorder, and bipolar II disorder), and anxiety disorder (panic disorder with or without agoraphobia, agoraphobia without a history of panic disorder, social phobia, specific phobia, generalized anxiety disorder, posttraumatic stress disorder, and separation anxiety disorder) diagnoses according to criteria from the Diagnostic and Statistical Manual of Mental Disorders, Fourth Edition (DSM-IV) [31] were used in the current study. Due to an error in skip logic, obsessive-compulsive disorder data were not valid and, therefore, were not used in the present study. Diagnostic hierarchy rules and organic exclusion rules were used in making all diagnoses.

Good concordance was found between CIDI diagnoses and diagnoses based on the Structured Clinical Interview for DSM-IV (SCID) [32] in a probability subsample of NCS-R participants for mood and anxiety disorders [33, 34]. The diagnostic algorithms used for eating disorders in the NCS-R have been reported elsewhere [18]; most of the CIDI questions closely aligned with the DSM-IV criteria for each eating disorder. However, there were two notable differences: (1) the loss-of-control aspect of binge-eating episodes required for DSM-IV diagnoses of bulimia nervosa and binge-eating disorder was not assessed directly by the CIDI, but rather indirectly with questions about attitudes and behaviors indicating loss of control (e.g., "Did you usually continue to eat even when you didn't feel hungry?") and distress (e.g., "Did you often get upset both during and after binges that your eating was out of your control?"), and (2) the CIDI assesses regular binge-eating episodes for three months or longer, whereas DSM-IV criteria require regular binge-eating episodes for 6 months or longer for a diagnosis of binge-eating disorder [18]. Despite these differences, previously published sensitivity analyses using stricter criteria for bulimia nervosa and binge-eating disorder in this sample yielded no substantial differences in prevalence estimates [18]. No 12-month cases of anorexia nervosa were detected in the NCS-R sample, precluding the ability to examine anorexia nervosa in the present study.

\section{Covariates}

Age (continuous), sex (dichotomous: male or female), structurally racialized categories labelled as race/ethnicity (categorical: non-Hispanic White, non-Hispanic Black, Hispanic/Latinx, or other), education (categorical: $<12$ years,
12 years, $13-15$ years, or $\geq 16$ years of education completed), and income-to-poverty ratio (continuous) were examined as sociodemographic covariates. Using self-reported household income and household size, as well as 2001 Census guidelines [35], income-to-poverty ratio was calculated as household income divided by the poverty threshold for household size.

\section{Statistical analysis}

Analyses were conducted with Stata 16.0, accounted for the complex sampling design, and incorporated weights to account for oversampling of participants meeting criteria for a mental disorder in the Part I sample, differential probabilities of selection within households, non-response bias, and differences between the sample and the 2000 Census with regard to sociodemographic and geographic characteristics [29]. Descriptive statistics were calculated, and $t$ tests and chi-square tests with Bonferroni adjustments were used to examine differences in sociodemographic characteristics and categories of 12-month DSM-IV disorders by food security status. Associations between 12-month food insecurity and 12-month DSM-IV disorders were examined using modified Poisson regression models (i.e., with robust standard errors) to calculate prevalence ratios [36]. In primary analyses, we conducted separate regression models for each combined category representing eating disorders (hereafter referred to as bulimic-spectrum eating disorders as there were no cases of anorexia nervosa), mood disorders, and anxiety disorders. In secondary analyses, we conducted separate regression models for each specific DSM-IV disorder to assess for consistency of observed associations within each category. All regression models were adjusted for age, sex, race/ethnicity, education, and income-to-poverty ratio. Weight status was considered for inclusion as a covariate but was ultimately excluded from the models because literature suggests it may act as a mediator (i.e., on the causal pathway from food insecurity to mental disorders) or collider (i.e., common effect of both food insecurity and mental disorders) [37], in which case adjustment for weight status could introduce overadjustment bias or selection bias, respectively.

\section{Results}

This sample of adults (mean [standard error] age $=44.9[0.5]$ years) was $46.6 \%$ male and $53.4 \%$ female, and the racial/ ethnic composition was $74.3 \%$ non-Hispanic White, $11.5 \%$ non-Hispanic Black, $10.2 \%$ Hispanic/Latinx, and $4.0 \%$ other. Over the 12 months prior to data collection, $11.1 \%$ of participants in this sample had experienced food insecurity. Using 12-month DSM-IV criteria, $1.5 \%$ of participants met criteria for a bulimic-spectrum eating disorder, $8.7 \%$ met criteria 
for a mood disorder, and $\mathbf{1 9 . 0 \%}$ met criteria for an anxiety disorder. Differences in sociodemographic characteristics and each category of 12-month DSM-IV disorder by food security status are reported in Table 1.

Cross-sectional associations between food insecurity and each category of 12-month DSM-IV disorder are presented in Fig. 1. After adjusting for sociodemographic characteristics, food insecurity was significantly associated with greater prevalence of bulimic-spectrum eating disorders (prevalence ratio $[\mathrm{PR}]=3.81 ; 95 \%$ confidence interval $[\mathrm{CI}] 2.26,6.42)$, mood disorders ( $\mathrm{PR}=2.53$; $95 \%$ CI 1.96, 3.29), and anxiety disorders ( $P R=1.69 ; 95 \%$ CI $1.39,2.07)$. Non-overlapping confidence intervals indicate that the association for bulimic-spectrum eating disorders was significantly stronger than the association for anxiety disorders. As shown in Table 2, food insecurity was significantly associated with each specific 12-month DSM-IV disorder examined except for bipolar I disorder and generalized anxiety disorder.
Table 1 Participant characteristics by food security status in a subsample of U.S. adults in the National Comorbidity Survey Replication II

\begin{tabular}{llll}
\hline & Food secure $(N=2554)$ & $\begin{array}{l}\text { Food insecure } \\
(N=360)\end{array}$ & $p$ \\
\hline Age in years, M (SE) & $45.3(0.5)$ & $41.8(1.4)$ & 0.02 \\
Sex, $\%(n)$ & & & \\
Male & $46.9(1,069)$ & $44.5(129)$ & 0.52 \\
Female & $53.1(1,485)$ & $55.5(231)$ & \\
Race/ethnicity, $\%(n)$ & & & \\
Non-Hispanic White & $75.3(1,945)^{\mathrm{a}}$ & \\
Non-Hispanic Black & $11.3(287)^{\mathrm{a}}$ & $66.0(232)^{\mathrm{b}}$ & \\
Hispanic/Latinx & $9.9(213)^{\mathrm{a}}$ & $13.7(57)^{\mathrm{a}}$ & \\
Other & $3.6(109)^{\mathrm{a}}$ & $7.5(30)^{\mathrm{b}}$ & \\
Years of education completed, $\%(n)$ & & $19.5(83)^{\mathrm{b}}$ & 0.003 \\
$<12$ & $14.4(332)^{\mathrm{a}}$ & $38.9(112)^{\mathrm{b}}$ & \\
12 & $31.7(747)^{\mathrm{a}}$ & $26.3(111)^{\mathrm{a}}$ & \\
$13-15$ & $28.9(790)^{\mathrm{a}}$ & $15.2(54)^{\mathrm{b}}$ & \\
$\geq 16$ & $25.0(685)^{\mathrm{a}}$ & $2.8(0.2)$ & $<0.001$ \\
Income-to-poverty ratio, M (SE) & $4.6(0.1)$ & $4.5(25)$ & $<0.001$ \\
12-month DSM-IV disorders, $\%(n)$ & & $21.0(105)$ & $<0.001$ \\
Bulimic-spectrum eating disorder & $1.2(41)$ & $32.4(167)$ & $<0.001$ \\
Mood disorder & $7.1(313)$ & & \\
Anxiety disorder & $17.4(713)$ & & \\
\hline
\end{tabular}

Statistics are weighted to account for the complex sampling design and non-response, with the exception of $n$ 's, which represent observed counts. Within rows, different superscript letters indicate statistically significant differences between food-secure and food-insecure participants after Bonferroni corrections

$M$ mean, $S E$ standard error

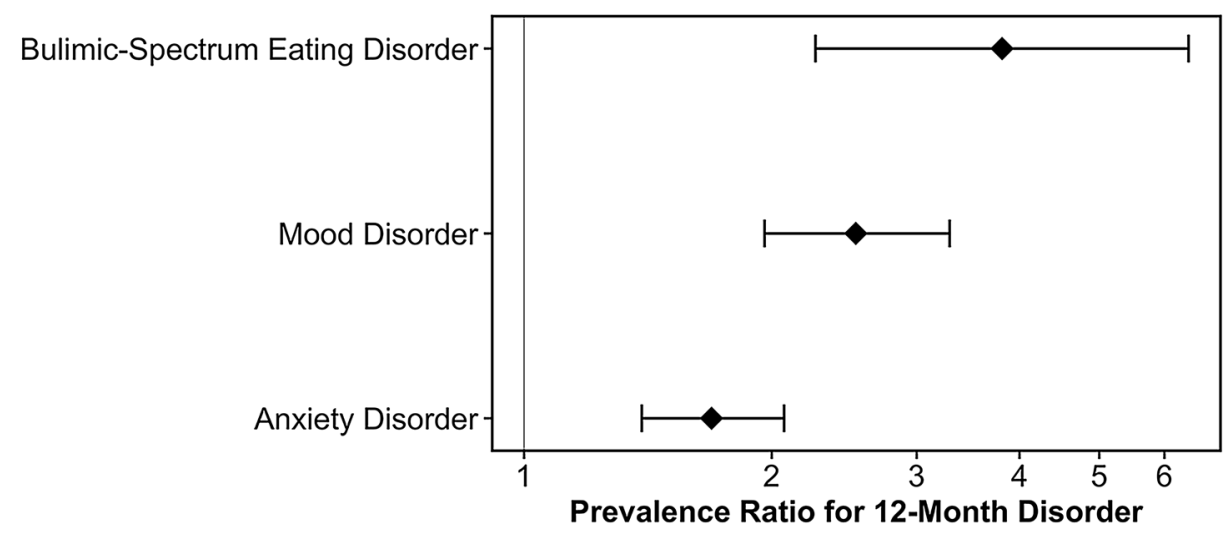

Note. Models were adjusted for age, sex, race/ethnicity, education, and income-to-poverty ratio. Error bars indicate $95 \%$ confidence intervals.
Fig. 1 Prevalence ratios for each category of 12-month DSM-IV disorder, comparing foodinsecure versus food-secure individuals in a subsample of U.S. adults in the National Comorbidity Survey Replication II 
Table 2 Prevalence ratios for specific 12-month DSM-IV disorders, comparing foodinsecure versus food-secure individuals in a subsample of U.S. adults in the National Comorbidity Survey Replication II

\begin{tabular}{lll}
\hline & $\begin{array}{l}\%(n) \text { with each disorder in full } \\
\text { sample }\end{array}$ & PR $(95 \%$ CI $)$ \\
\hline $\begin{array}{l}\text { Bulimic-spectrum eating disorders } \\
\text { Bulimia nervosa }\end{array}$ & $0.3(16)$ & $4.80(1.64,13.99)^{* *}$ \\
Binge-eating disorder & $1.2(50)$ & $3.49(1.84,6.62)^{* * *}$ \\
Mood disorders & & \\
Major depressive disorder & $7.0(338)$ & $2.62(2.01,3.43)^{* * *}$ \\
Dysthymia & $1.7(74)$ & $2.02(1.18,3.46)^{*}$ \\
Bipolar I disorder & $0.7(35)$ & $2.05(0.84,4.97)$ \\
Bipolar II disorder & $0.8(37)$ & $3.63(1.47,8.99)^{* *}$ \\
Anxiety disorders & & \\
Panic disorder & $2.8(136)$ & $2.75(1.88,4.02)^{* * *}$ \\
Agoraphobia without panic & $1.3(65)$ & $3.17(1.22,8.21)^{*}$ \\
Specific phobia & $9.3(432)$ & $1.97(1.54,2.51)^{* * *}$ \\
Social phobia & $7.1(342)$ & $2.10(1.41,3.13)^{* *}$ \\
Generalized anxiety disorder & $2.8(134)$ & $1.30(0.75,2.23)$ \\
Posttraumatic stress disorder & $3.4(159)$ & $3.33(2.11,5.24)^{* * *}$ \\
Separation anxiety disorder & $2.2(83)$ & $3.46(1.62,7.41)^{* *}$ \\
\hline
\end{tabular}

Models were adjusted for age, sex, race/ethnicity, education, and income-to-poverty ratio

$P R$ prevalence ratio, $C I$ confidence interval

${ }^{*} p<0.05, * * p<0.01, * * * p<0.001$

\section{Discussion}

In this nationally representative sample of U.S. adults, 12-month diagnoses of bulimic-spectrum disorders, mood disorders, and anxiety disorders were more common among individuals who had experienced food insecurity than among those who were food secure, with the greatest difference observed for bulimic-spectrum eating disorders. Observed differences held even when accounting for education and income-to-poverty ratio as indicators of socioeconomic status, suggesting a unique role of food insecurity above and beyond that of socioeconomic status broadly. Overall, the results of this study provide evidence in support of crosssectional associations among adults between food insecurity and mental disorders across eating, mood, and anxiety domains.

Results of the present study corroborate a growing body of evidence indicating a link between food insecurity and eating disorders. While prior work in a sample of adults recruited online has demonstrated greater prevalence of bulimia nervosa [17] and binge-eating disorder [16] as assessed via self-administered questionnaire among those living in food-insecure households, the present study is the first, to our knowledge, to confirm these findings within a sample from which results can be generalized to the U.S. population with some confidence, as well as to confirm them with face-to-face diagnostic interview data. Faceto-face diagnostic interviews have many advantages over self-administered surveys. In addition to yielding higher participation rates and less item-level missing data than selfadministered surveys [38], face-to-face interviews allow for interviewers to gauge respondent fatigue and suggest breaks accordingly, thereby helping respondents maintain adequate concentration throughout the interview [29]. Moreover, compared to self-administered surveys, face-to-face interviews offer the benefits of less recall bias [38], less likelihood of responses being biased by a list of response options [38], and the opportunity for interviewers to probe for thoughtful, clear, and thorough answers [26]. In addition, although the depression and anxiety literatures on food insecurity are more extensive than the eating disorders literature on food insecurity, the present study is the first that we are aware of to utilize diagnostic interview data from a nationally representative sample to examine food insecurity's associations with mood and anxiety disorders among adults, as well as the first to examine its associations with specific mood and anxiety disorders. Using data from the NCS-R's parallel study among adolescents, the National Comorbidity Survey Replication Adolescent Supplement (NCS-A), McLaughlin and colleagues found 12-month mood and anxiety disorders to be more common among adolescents living in foodinsecure households than among those living in food-secure households [9]. The mood and anxiety disorder findings from the present study closely mirror those from the study by McLaughlin and colleagues [9], suggesting that the link between food insecurity and mood and anxiety disorders is relevant across both adolescent and adult life stages. However, because McLaughlin and colleagues examined mood 
and anxiety disorders only as overall categories (i.e., any mood disorder, any anxiety disorder), their study is not able to help contextualize the null findings for bipolar I disorder and generalized anxiety disorder in the present study. While the null finding for bipolar I disorder may have been related to low statistical power, the null finding for generalized anxiety disorder was particularly surprising considering that worry is a core symptom of generalized anxiety disorder [31] and appears to be a common theme among individuals living with food insecurity [13]. More research is, therefore, needed to better understand the relationship between food insecurity and generalized anxiety disorder.

In addition to its methodological strengths, the present study has important limitations to consider. Although this study aimed to examine anorexia nervosa, bulimia nervosa, and binge-eating disorder in relation to food insecurity, the sample had no cases of 12-month anorexia nervosa, precluding our ability to investigate the association with anorexia nervosa. No studies to date have examined the relationship between food insecurity and anorexia nervosa; therefore, because we were unable to examine this relationship in the present study, this remains a critical gap in the literature. Additional limitations of the present study pertain to measurement. Although food insecurity was assessed using a measure derived from the Six-Item Short Form U.S. Household Food Security Survey Module [30], items were modified and skip logic was used to reduce participant burden. These changes may have resulted in misclassification of food insecurity for some participants. With regard to measurement of eating disorders, discrepancies between the CIDI questions and the DSM-IV criteria for bulimia nervosa and binge-eating disorder may have also resulted in some misclassification. Notably, the use of DSM-IV diagnostic criteria likely resulted in underestimates of bulimia nervosa, binge-eating disorder, and anorexia nervosa as compared to DSM-5 diagnostic criteria [39-41], and it is possible that participants who did not meet the more stringent DSM-IV criteria may differ with respect to food security status. However, assuming a true prevalence ratio greater than one, our results are expected to be biased toward the null (i.e., demonstrating weaker associations than they would otherwise) if misclassification of eating disorder diagnoses due to using DSM-IV criteria either (a) did not differ by food security status or (b) was more likely to underestimate eating disorders among food-insecure participants than among food-secure participants [42, 43]. Only if eating disorders were more likely to be underestimated among food-secure participants than among food-insecure participants using DSM-IV criteria would our results be expected to potentially demonstrate bias away from the null (i.e., demonstrating stronger associations than they would otherwise) [43]. Another limitation related to measurement is that an error in skip logic precluded our ability to examine obsessive-compulsive disorder. Further, all mental disorders were assessed according to DSM-IV criteria in this study rather than the current DSM-5 criteria, reflecting the age of the NCS-R data, which represents another important limitation of the present study. As data were collected in 2001-2003, it is possible that the associations observed in the present study may not be generalizable to current times. While the prevalence of food insecurity in the present study was very similar to that observed in the U.S. population in 2019 [2], further research is needed to better understand the relationship between food insecurity in the post-COVID-19 economic context and eating disorders as defined by the more inclusive DSM- 5 diagnostic criteria. Finally, the cross-sectional nature of the data is a key limitation of this study, as it precludes the ability to make any temporal inferences, and very little longitudinal research has been conducted on this topic. The limited existing longitudinal literature in the depression and anxiety fields suggests a bidirectional relationship with food insecurity [8], but no studies to date have offered any clarity on the temporality of the relationship between food insecurity and eating disorders. Therefore, longitudinal research in this area is eminently needed.

While more research is needed to elucidate the temporality of the associations observed in this study, results emphasize the need to expand intervention efforts to reduce food insecurity and to improve access to mental health services for food-insecure populations. Given the high rates of comorbidity between eating disorders, mood disorders, and anxiety disorders, as well as the link between food insecurity and disorders in each of these domains, intervening on each of these targets simultaneously may result in improved efficiency and dissemination reach of interventions. Importantly, because food insecurity disproportionately affects racial/ethnic minorities [2], these interventions must also address the underlying systemic inequities. Approaches we must take to work toward eliminating racial/ethnic disparities in food insecurity were recently outlined: we must elevate the voices of those affected by food insecurity in our research, we must utilize trauma-informed approaches, and we must incite change not only in policies that clearly pertain to health, but also in those which address upstream factors such as housing, education, employment, and criminal justice [44].

Supplementary Information The online version contains supplementary material available at https://doi.org/10.1007/s00127-021-02126-5.

Funding The National Comorbidity Survey Replication (NCS-R) was supported by the National Institute of Mental Health (Grant Number U01MH60220) with supplemental support from the National Institute of Drug Abuse, the Substance Abuse and Mental Health Services Administration, the Robert Wood Johnson Foundation (Grant Number 044780), Eli Lilly and Company, and the John W. Alden Trust. Preparation of this article was supported in part by the National Institute of Mental Health (Grant Number T32MH082761). 
Data availability The data that support the findings of this study are publicly available at https://www.icpsr.umich.edu/web/ICPSR/studies/ 20240 .

Code availability The code used for the analyses in this study is available from the corresponding author upon request.

\section{Declarations}

Conflict of interest The authors declare that they have no conflicts of interest.

\section{References}

1. Anderson SA (1990) Core indicators of nutritional state for difficult-to-sample populations. J Nutr 120(11):1555-1600

2. Coleman-Jensen A, Rabbitt MP, Gregory CA, Singh A (2020) Household food security in the United States in 2019. ERR-275, U.S. Department of Agriculture, Economic Research Service

3. Waxman E, Gupta P, Karpman M (2020) More than one in six adults were food insecure two months into the COVID-19 recession. Urban Institute

4. Hanson KL, Connor LM (2014) Food insecurity and dietary quality in US adults and children: a systematic review. Am J Clin Nutr 100:684-692. https://doi.org/10.3945/ajcn.114.084525.1

5. Weaver LJ, Fasel CB (2018) A systematic review of the literature on the relationships between chronic diseases and food insecurity. Food Nutr Sci 9:519-541. https://doi.org/10.4236/fns.2018.95040

6. Arenas DJ, Thomas A, Wang JC, DeLisser HM (2019) A systematic review and meta-analysis of depression, anxiety, and sleep disorders in US adults with food insecurity. J Gen Intern Med 34:2874-2882. https://doi.org/10.1007/s11606-019-05202-4

7. Myers CA (2020) Food insecurity and psychological distress: a review of the recent literature. Curr Nutr Rep 9:107-118. https:// doi.org/10.1007/s13668-020-00309-1

8. Bruening M, Dinour LM, Chavez JBR (2017) Food insecurity and emotional health in the USA: a systematic narrative review of longitudinal research. Public Health Nutr 20:3200-3208. https:// doi.org/10.1017/S1368980017002221

9. McLaughlin KA, Green JG, Alegría M et al (2012) Food insecurity and mental disorders in a national sample of U.S. adolescents. J Am Acad Child Adolesc Psychiatry 51:1293-1303. https://doi. org/10.1016/j.jaac.2012.09.009

10. Muldoon KA, Duff PK, Fielden S, Anema A (2013) Food insufficiency is associated with psychiatric morbidity in a nationally representative study of mental illness among food insecure Canadians. Soc Psychiatry Psychiatr Epidemiol 48:795-803. https:// doi.org/10.1007/s00127-012-0597-3

11. Pryor L, Lioret S, van der Waerden J et al (2016) Food insecurity and mental health problems among a community sample of young adults. Soc Psychiatry Psychiatr Epidemiol 51:1073-1081. https:// doi.org/10.1007/s00127-016-1249-9

12. Swales S, May C, Nuxoll M, Tucker C (2020) Neoliberalism, guilt, shame and stigma: a Lacanian discourse analysis of food insecurity. J Community Appl Soc Psychol 30:673-687. https://doi.org/ 10.1002/casp. 2475

13. Meza A, Altman E, Martinez S, Leung CW (2019) "It's a feeling that one is not worth food": a qualitative study exploring the psychosocial experience and academic consequences of food insecurity among college students. J Acad Nutr Diet 119:1713-1721. e1. https://doi.org/10.1016/j.jand.2018.09.006
14. Becker CB, Middlemass K, Taylor B et al (2017) Food insecurity and eating disorder pathology. Int J Eat Disord 50:1031-1040. https://doi.org/10.1002/eat.22735

15. Becker CB, Middlemass KM, Gomez F, Martinez-Abrego A (2019) Eating disorder pathology among individuals living with food insecurity: a replication study. Clin Psychol Sci 7:11441158. https://doi.org/10.1177/2167702619851811

16. Rasmusson G, Lydecker JA, Coffino JA et al (2019) Household food insecurity is associated with binge-eating disorder and obesity. Int J Eat Disord 52:28-35. https://doi.org/10.1002/eat.22990

17. Lydecker JA, Grilo CM (2019) Food insecurity and bulimia nervosa in the United States. Int J Eat Disord 52:735-739. https:// doi.org/10.1002/eat.23074

18. Hudson JI, Hiripi E, Pope HG, Kessler RC (2007) The prevalence and correlates of eating disorders in the National Comorbidity Survey Replication. Biol Psychiatry 61:348-358. https://doi.org/ 10.1016/j.biopsych.2006.03.040

19. Deloitte Access Economics (2020) Social and economic cost of eating disorders in the United States of America: Report for the strategic training initiative for the prevention of eating disorders and the academy for eating disorders

20. Mitchell JE, Crow S (2006) Medical complications of anorexia nervosa and bulimia nervosa. Curr Opin Psychiatry 19:438-443. https://doi.org/10.1097/01.yco.0000228768.79097.3e

21. Swanson SA, Crow SJ, Le Grange D et al (2011) Prevalence and correlates of eating disorders in adolescents: results from the National Comorbidity Survey Replication Adolescent Supplement. Arch Gen Psychiatry 68:714-723. https://doi.org/10.1001/ archgenpsychiatry.2011.22

22. Gard MCE, Freeman CP (1996) The dismantling of a myth: a review of eating disorders and socioeconomic status. Int J Eat Disord 20:1-12. https://doi.org/10.1002/(SICI)1098-108X(199607) 20:1\%3c1::AID-EAT1\%3e3.0.CO;2-M

23. Larson N, Laska MN, Neumark-Sztainer D (2020) Food insecurity, diet quality, home food availability, and health risk behaviors among emerging adults: findings from the EAT 2010-2018 study. Am J Public Health 110:1422-1428. https://doi.org/10.2105/ AJPH.2020.305783

24. Bruening M, MacLehose R, Loth K et al (2012) Feeding a family in a recession: food insecurity among Minnesota parents. Am J Public Health 102:520-526. https://doi.org/10.2105/AJPH.2011. 300390

25. Hooper L, Telke S, Larson N et al (2020) Household food insecurity: associations with disordered eating behaviours and overweight in a population-based sample of adolescents. Public Health Nutr 23:3126-3135. https://doi.org/10.1017/S1368980020000464

26. Kessler RC, Üstün BB (2004) The World Mental Health (WMH) Survey Initiative version of the World Health Organization (WHO) Composite International Diagnostic Interview (CIDI). Int J Methods Psychiatr Res 13:93-117. https://doi.org/10.1002/ mpr. 168

27. Udo T, Grilo CM (2018) Prevalence and correlates of DSM-5defined dating disorders in a nationally representative sample of U.S. adults. Biol Psychiatry 84:345-354. https://doi.org/10.1016/j. biopsych.2018.03.014

28. Kessler RC, Merikangas KR (2004) The National Comorbidity Survey Replication (NCS-R): background and aims. Int J Methods Psychiatr Res 13:60-68. https://doi.org/10.1002/mpr.166

29. Kessler RC, Berglund P, Chiu WT et al (2004) The US National Comorbidity Survey Replication (NCS-R): design and field procedures. Int J Methods Psychiatr Res 13:69-92. https://doi.org/10. 1002/mpr.167

30. Blumberg SJ, Bialostosky K, Hamilton WL, Briefel RR (1999) The effectiveness of a short form of the household food security scale. Am J Public Health 89:1231-1234. https://doi.org/10.2105/ AJPH.89.8.1231 
31. American Psychiatric Association (1994) Diagnostic and statistical manual of mental disorders, fourth edition (DSM-IV). American Psychiatric Association, Washington, DC

32. First MB, Spitzer RL, Gibbon M, Williams JB (2002) Structured clinical interview for DSM-IV-TR Axis I disorders, research version, non-patient edition. Biometrics Research, New York State Psychiatric Institute, New York

33. Kessler RC, Abelson J, Demler O et al (2004) Clinical calibration of DSM-IV diagnoses in the World Mental Health (WMH) version of the World Health Organization (WHO) Composite International Diagnostic Interview (WMH-CIDI). Int J Methods Psychiatr Res 13:122-139. https://doi.org/10.1002/mpr.169

34. Kessler RC, Berglund P, Demler O et al (2005) Lifetime prevalence and age-of-onset distributions of DSM-IV disorders in the National Comorbidity Survey Replication. Arch Gen Psychiatry 62:593-602. https://doi.org/10.1001/archpsyc.62.6.593

35. Proctor BD, Dalaker J (2002) Current Population Reports. In: poverty in the United States: 2001. U.S. Government Printing Office, Washington, DC

36. Barros AJD, Hirakata VN (2003) Alternatives for logistic regression in cross-sectional studies: an empirical comparison of models that directly estimate the prevalence ratio. BMC Med Res Methodol 3:1-13. https://doi.org/10.1186/1471-2288-3-21

37. Cheung HC, Shen A, Oo S et al (2015) Food insecurity and body mass index: a longitudinal mixed methods study, Chelsea, Massachusetts, 2009-2013. Prev Chronic Dis 12:1-13. https://doi.org/ $10.5888 / \operatorname{pcd} 12.150001$
38. Bowling A (2005) Mode of questionnaire administration can have serious effects on data quality. J Public Health (Bangkok) 27:281-291. https://doi.org/10.1093/pubmed/fdi031

39. Call C, Walsh BT, Attia E (2013) From DSM-IV to DSM-5: changes to eating disorder diagnoses. Curr Opin Psychiatry 26:532-536. https://doi.org/10.1097/YCO.0b013e328365a321

40. Mancuso SG, Newton JR, Bosanac P et al (2015) Classification of eating disorders: comparison of relative prevalence rates using DSM-IV and DSM-5 criteria. Br J Psychiatry 206:519-520. https://doi.org/10.1192/bjp.bp.113.143461

41. Vo M, Accurso EC, Goldschmidt AB, Le Grange D (2017) The impact of DSM-5 on eating disorder diagnoses. Int J Eat Disord 50:578-581. https://doi.org/10.1002/eat.22628

42. Copeland KT, Checkoway H, Mcmichael AJ, Holbrook RH (1977) Bias due to misclassification in the estimation of relative risk. Am J Epidemiol 105:488-495. https://doi.org/10.1093/oxfordjournals. aje.a112408

43. Chen Q, Galfalvy H, Duan N (2013) Effects of disease misclassification on exposure-disease association. Am J Public Health 103:67-74. https://doi.org/10.2105/AJPH.2012.300995

44. Raskind IG (2020) Hunger does discriminate: addressing structural racism and economic inequality in food insecurity research. Am J Public Health 110:1264-1265. https://doi.org/10.2105/ AJPH.2020.305841 